\title{
Double Polarized Neutron-Proton Scattering and Meson-Exchange Nucleon-Nucleon Potential Models
}

\author{
B. W. Raichle, ${ }^{1,4}$ C. R. Gould, ${ }^{2,4}$ D. G. Haase, ${ }^{2,4}$ M. L. Seely, ${ }^{2,4, *}$ J. R. Walston, ${ }^{2,4, \dagger}$ W. Tornow, ${ }^{3,4}$ W. S. Wilburn, ${ }^{3,4,+}$ \\ S. I. Penttilä, ${ }^{5}$ and G. W. Hoffmann ${ }^{6}$ \\ ${ }^{1}$ Department of Physical Sciences, Morehead State University, Morehead, Kentucky 40351 \\ ${ }^{2}$ Physics Department, North Carolina State University, Raleigh, North Carolina 27695 \\ ${ }^{3}$ Physics Department, Duke University, Durham, North Carolina 27708 \\ ${ }^{4}$ Triangle Universities Nuclear Laboratory, Durham, North Carolina 27708 \\ ${ }^{5}$ Los Alamos National Laboratory, Los Alamos, New Mexico 87545 \\ ${ }^{6}$ University of Texas, Austin, Texas 78712
}

(Received 17 February 1999)

\begin{abstract}
We report on polarized beam-polarized target measurements of the spin-dependent neutron-proton total cross-section differences in longitudinal and transverse geometries ( $\Delta \sigma_{L}$ and $\Delta \sigma_{T}$, respectively) between $E_{n}=5$ and $20 \mathrm{MeV}$. Single-parameter phase-shift analyses were performed to extract the phase-shift mixing parameter $\varepsilon_{1}$, which characterizes the strength of the nucleon-nucleon tensor interaction at low energies. Consistent with the trend of previous determinations at $E_{n}=25$ and $50 \mathrm{MeV}$, our values for $\varepsilon_{1}$ imply a stronger tensor force than predicted by meson-exchange nucleonnucleon potential models and nucleon-nucleon phase-shift analyses.
\end{abstract}

PACS numbers: 25.40.Dn, 13.75.Cs, 24.70.+s, 25.10.+s

The recent meson-exchange (ME) based models of the nucleon-nucleon $(N N)$ interaction [1-3] have achieved remarkable success in describing $N N$ scattering data and a large body of nuclear physics phenomena [4]. The description of the long-range component of the $N N$ force in terms of pion exchange is especially compelling. Nevertheless, when these models are used as input into exact calculations of few-body systems, a few, but persistent and well documented, discrepancies arise, for example, the underbinding of the three-body bound-state systems [5] and the underprediction of the nucleon-deuteron analyzing power at low energies [6]. While most attempts to explain these discrepancies have focused on three-body force effects, small deficiencies in the current parametrization of the $N N$ interaction remain a remote source of possible explanations.

In this paper we take advantage of advances in polarized beam and polarized target technology to test the description of the $N N$ interaction at considerably improved accuracy. Specifically, we investigate the $N N$ tensor force at low energies, for which the ME mechanism provides a precise prediction [7]. The associated ${ }^{3} S_{1-}{ }^{3} D_{1}$ mixing parameter $\varepsilon_{1}$ is theoretically well understood and, unless the current description of ME is abandoned, there is little room for any sizable modification of $\varepsilon_{1}$ values at low energies [3]. Therefore, among the possible test cases for probing the accuracy of the present description of the $N N$ interaction, the comparison of experimental data and $N N$ potentialmodel predictions for $\varepsilon_{1}$ provides a unique opportunity to observe small deviations from ME based predictions.

The data come from the TUNL (Triangle Universities Nuclear Laboratory) measurements of the spin-dependent total cross-section differences $\Delta \sigma_{L}$ and $\Delta \sigma_{T}$ for a polarized neutron beam transmitted through a polarized proton target in longitudinal $(L)$ geometry (spins parallel to the neutron beam momentum) and transverse $(T)$ geometry (spins perpendicular to the neutron beam momentum):

$$
\begin{gathered}
\Delta \sigma_{L}=\sigma(\rightleftarrows)-\sigma(\rightrightarrows), \\
\Delta \sigma_{T}=\sigma(\uparrow \downarrow)-\sigma(\uparrow \uparrow) .
\end{gathered}
$$

These measurements have allowed the determination of the observable $\Delta=\Delta \sigma_{L}-\Delta \sigma_{T}$ which provides a very sensitive and practically unique measure of the lowenergy mixing parameter $\varepsilon_{1}$.

The transmitted flux $\Phi$ of a neutron beam with polarization $P_{n}$ incident on a proton target with polarization $P_{T}$ is the weighted sum of the transmission of a beam of pure spin-up neutrons $\left(P_{n}=1\right)$ plus the transmission of a beam of pure spin-down neutrons $\left(P_{n}=-1\right)$, and is given by

$$
\Phi=\Phi_{0}^{+}\left[e^{-N_{p}^{+} x \sigma_{p}} e^{-N_{p}^{-} x \sigma_{a}}\right]+\Phi_{0}^{-}\left[e^{-N_{p}^{+} x \sigma_{a}} e^{-N_{p}^{-} x \sigma_{p}}\right],
$$

where the fraction of spin-up $\left(N_{p}^{+}\right)$or spin-down $\left(N_{p}^{-}\right)$ protons is given by $N_{p}^{ \pm}=\frac{1}{2}\left(1 \pm P_{T}\right)$ and the number of incident spin-up $\left(\Phi_{0}^{+}\right)$or spin-down $\left(\Phi_{0}^{-}\right)$neutrons is similarly given in terms of $P_{n}$. The quantity $x$ is the target thickness in units of $\mathrm{b}^{-1}$, and $\sigma_{p}\left(\sigma_{a}\right)$ are the total cross sections for neutrons and protons with spin axes parallel (antiparallel), by convention defined as

$$
\begin{aligned}
& \sigma_{p}=\sigma_{0}-\frac{1}{2} \Delta \sigma_{L(T)}, \\
& \sigma_{a}=\sigma_{0}+\frac{1}{2} \Delta \sigma_{L(T)},
\end{aligned}
$$

where $\sigma_{0}$ is the unpolarized total cross section. 
The transmission asymmetry $\epsilon_{n}$ due to reversing the neutron beam polarization from $-P_{n}^{\prime \prime}$ to $+P_{n}^{\prime}$ is defined as

$$
\epsilon_{n}=\frac{\Phi\left(+P_{n}^{\prime}\right)-\Phi\left(-P_{n}^{\prime \prime}\right)}{\Phi\left(+P_{n}^{\prime}\right)+\Phi\left(-P_{n}^{\prime \prime}\right)}
$$

which can be rewritten as

$$
\boldsymbol{\epsilon}_{n}=\frac{\left(P_{n}^{\prime}+P_{n}^{\prime \prime}\right) \sinh (y)}{2 \cosh (y)+2\left(P_{n}^{\prime}-P_{n}^{\prime \prime}\right) \sinh (y)},
$$

using the shorthand notation $y=\frac{1}{2} P_{T} x \Delta \sigma_{L(T)}$.

The polarized proton targets used in our measurements meet the criterion $y<0.02 \ll 1$ and the TUNL polarized ion source [8] is such that $P_{n}^{\prime} \approx P_{n}^{\prime \prime}=P_{n}$ so that Eq. (7) becomes

$$
\epsilon_{n}=\frac{1}{2} P_{n} P_{T} x \Delta \sigma_{L(T)} .
$$

Notably, the transmission asymmetry is independent of the incident neutron flux and the unpolarized total cross section.

The proton target polarization was achieved via dynamic nuclear polarization (DNP) [9]. The target material was propanediol $\left[\mathrm{C}_{3} \mathrm{H}_{6}(\mathrm{OH})_{2}\right.$, density $\left.1.27 \mathrm{~g} / \mathrm{cm}^{3}\right]$ frozen into $1 \mathrm{~mm}$ diam beads, giving a hydrogen density of $5 \times 10^{22} \mathrm{H} / \mathrm{cm}^{3}$. The propanediol was chemically doped with EHBA-Cr ${ }^{V}$ complex to provide free electrons for DNP. The nominal target thickness was $0.06 \mathrm{~b}^{-1}$ with a typical $P_{T}=65 \%$. The target beads filled a $1.4 \mathrm{~cm} \times 1.4 \mathrm{~cm} \times 1.4 \mathrm{~cm}$ cup. The target was cooled to $0.5 \mathrm{~K}$ in a $2.5 \mathrm{~T}$ magnetic field by a ${ }^{3} \mathrm{He}$ evaporation refrigerator [10]. The refrigerator has a cooling power of $15 \mathrm{~mW}$ at $0.5 \mathrm{~K}$ with a ${ }^{3} \mathrm{He}$ flow rate of $0.6 \mathrm{mmol} / \mathrm{s}$. Dynamic pumping was with $\approx 7 \mathrm{~mW}$ of $69 \mathrm{GHz}$ microwaves.

The polarized neutron beam was produced via the ${ }^{3} \mathrm{H}(\vec{p}, \vec{n})^{3} \mathrm{He}$ or ${ }^{2} \mathrm{H}(\vec{d}, \vec{n}){ }^{3} \mathrm{He}$ polarization-transfer reactions. The ${ }^{3} \mathrm{H}(\vec{p}, \vec{n})^{3} \mathrm{He}$ production target used at the lower energies was a $1.1 \mathrm{mg} / \mathrm{cm}^{2}$ thick layer of tritiated titanium deposited on a metallic beamstop. The ${ }^{2} \mathrm{H}(\vec{d}, \vec{n})^{3} \mathrm{He}$ production target used at the higher energies was a $6.0 \mathrm{~cm}$ long $\times 1.9 \mathrm{~cm}$ diameter gas cell filled to 3 atm deuterium gas.
A beam of polarized protons or deuterons was produced by the TUNL atomic beam polarized ion source. Typical proton or deuteron vector polarization was of order $60 \%$. Acceleration was provided by a $10 \mathrm{MV}$ FN tandem Van de Graaff accelerator. Typical beam current on target was of order $1 \mu \mathrm{A}$.

Neutrons were detected at $0^{\circ}$ by a $12.7 \mathrm{~cm}$ diameter $\times$ $12.7 \mathrm{~cm}$ long liquid organic scintillator. The $0^{\circ}$ detector was collimated to a $2.5 \mathrm{~ms}$ rad solid angle which illuminated a $1.2 \mathrm{~cm} \times 1.2 \mathrm{~cm}$ cross section of the target. The incident neutron flux was monitored by a small $(11.1 \mathrm{~mm} \times 22.2 \mathrm{~mm} \times 25.4 \mathrm{~mm}$ thick) liquid organic scintillator.

Figure 1 shows the experimental arrangement. The observables $\Delta \sigma_{L}$ or $\Delta \sigma_{T}$ can be determined from measurements of a neutron transmission asymmetry, neutron beam polarization, and the product of target polarization times thickness according to Eq. (8).

In practice, the measurement of a transmission asymmetry is susceptible to many systematic effects. Effects arising from beam polarization dependence of the neutron production yields, beam current asymmetries, dead-time correction asymmetries, and count-rate dependent neutron detector efficiencies have been carefully treated [11]. Time-ordered drifts were canceled to second order by reversing the neutron polarization at $10 \mathrm{~Hz}$ in the eight-step sequence +--+-++- . In addition, the target polarization was reversed every $4 \mathrm{~h}$.

The neutron beam polarization was determined to typically $3 \%-6 \%$ accuracy from measurements of the charged-particle beam polarization and the known polarization-transfer coefficients. The charged-particle beam polarization was determined by measuring a left and a right scattering asymmetry from $\vec{p}-{ }^{4} \mathrm{He}$ elastic scattering or from the ${ }^{3} \mathrm{He}(\vec{d}, p)$ reaction. The scattering chamber was located several meters upstream from the neutron production cell.

The proton target polarization times thickness was determined by neutron transmission at very low energies. Nuclear magnetic resonance was also used to continuously monitor the target polarization during the measurements. At very low energy $\Delta \sigma_{T(L)}$ is well determined

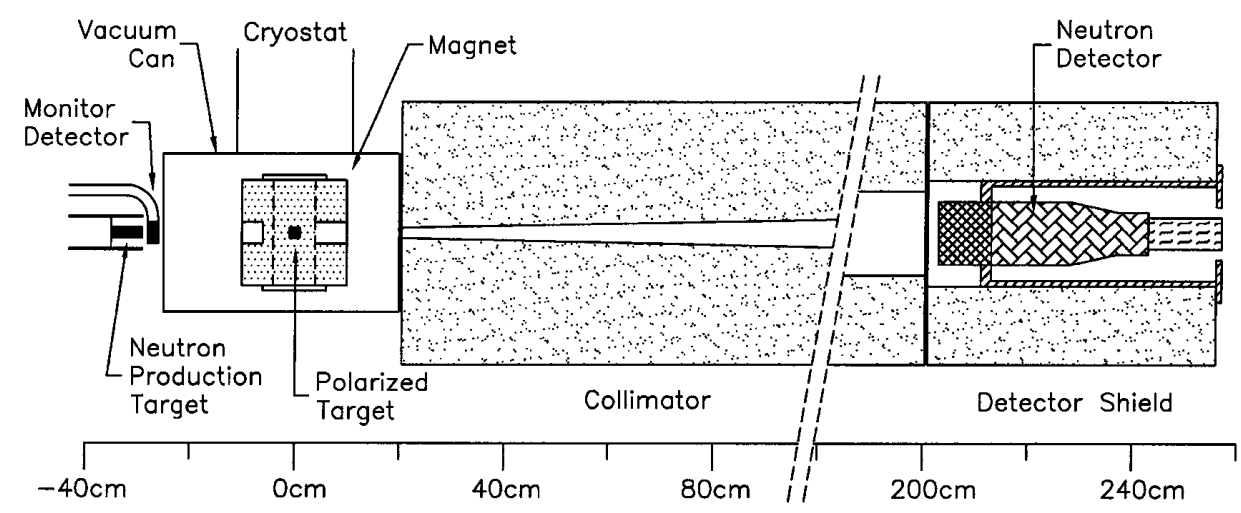

FIG. 1. Schematic of the experimental setup from the neutron production cell to the $0^{\circ}$ detector. 
TABLE I. Experimental values of $\Delta \sigma_{L}, \Delta \sigma_{T}$, and $\Delta$, and $\varepsilon_{1}$ results of the $\chi^{2}$ minimization of $\Delta_{\text {calc }}-\Delta_{\text {exp }}$. Energies are in MeV.

\begin{tabular}{ccccc}
\hline \hline$E_{n}$ & $\Delta \sigma_{L}(\mathrm{mb})$ & $\Delta \sigma_{T}(\mathrm{mb})$ & $\Delta(\mathrm{mb})$ & $\varepsilon_{1}\left(^{\circ}\right)$ \\
\hline 4.98 & $94.0 \pm 10.8$ & $41.0 \pm 33.8$ & $53.0 \pm 35.5$ & $0.38 \pm 0.26$ \\
7.43 & $-13.4 \pm 1.8^{\mathrm{a}}$ & $-129.0 \pm 11.9$ & $115.6 \pm 12.0$ & $1.16 \pm 0.12$ \\
10.7 & $-42.2 \pm 5.9$ & $-140.4 \pm 7.0$ & $98.2 \pm 9.2$ & $1.35 \pm 0.13$ \\
14.6 & $-51.5 \pm 4.6$ & $-143.0 \pm 7.2$ & $92.5 \pm 8.5$ & $1.69 \pm 0.16$ \\
17.1 & $-39.9 \pm 6.6$ & $-123.9 \pm 6.7$ & $84.0 \pm 9.4$ & $1.81 \pm 0.21$ \\
19.7 & $-32.8 \pm 7.2$ & $\cdots$ & $\cdots$ & $2.10 \pm 0.27$ \\
\hline \hline
\end{tabular}

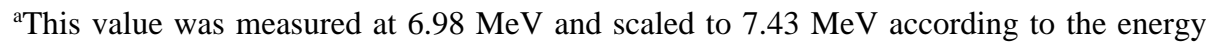
dependence of the Nijmegen partial wave PWA93 analysis [12].

by effective-range expansions, phase-shift analyses, and potential models, so Eq. (8) can be solved for $P_{T} x$ measuring $\epsilon_{n}$ and $P_{n}$. As the neutron energy goes to zero, the tensor interaction turns off and $\Delta \sigma_{T(L)}$ depends only upon the scalar spin-spin interaction, which, at zero energy, depends only on the singlet and triplet $n-p$ scattering lengths ${ }^{3} S_{1}$ and ${ }^{1} S_{0}$, respectively. As a result, all realistic calculations of $\Delta \sigma_{T(L)}$ agree to high precision at low neutron energies. The measurement was performed at $E_{n}=1.915$ (0.793) MeV for the transverse (longitudinal) geometry. The neutron beam energy was calibrated using ${ }^{12} \mathrm{C}$ resonances in neutron transmission experiments. For $\Delta \sigma_{T(L)}$ the average values using phase-shift analyses and potential models are 940 (3476) mb with a $\pm 2( \pm 8) \mathrm{mb}$ statistical standard deviation. For example, the Nijmegen partial wave PWA93 analysis [12] predicts 938 (3476) mb for $\Delta \sigma_{T(L)}$. Also the highly sophisticated effective-range expansion formulas of Mathelitsch and VerWest [13] provide results that are in excellent agreement with our average values. The total accuracy of our determination of $P_{T} x$ for the transverse and longitudinal geometries was $4 \%$ and $6 \%$, respectively.

Measurements of $\Delta \sigma_{T}$ were made between $E_{n}=11$ and $17 \mathrm{MeV}$ [14]. The $\Delta \sigma_{L}$ measurements covered an energy range from $E_{n}=5$ through $20 \mathrm{MeV}$ [15]. The results are listed in Table I. Error bars include both statistical and systematic uncertainties added in quadrature. These were about equal in magnitude. The 5 and $7 \mathrm{MeV}$ values of $\Delta \sigma_{T}$ are from Wilburn et al. [16].

The observable $\Delta=\Delta \sigma_{L}-\Delta \sigma_{T}$ is extremely sensitive to the phase-shift parameter $\varepsilon_{1}$, as shown in Fig. 2, and practically insensitive to other phase shifts which contribute to low-energy $n-p$ scattering [17]. Limiting ourselves for simplicity to $N N$ interactions with total angular momentum $J \leq 1, \Delta$ is explicitly given by

$$
\begin{aligned}
\Delta \approx \frac{2 \pi}{k^{2}}\{ & 2 \sin ^{2} \delta\left({ }^{3} P_{0}\right)-3 \sin ^{2} \delta\left({ }^{3} P_{1}\right) \\
& +3 \sin ^{2} \varepsilon_{1}+3 \cos 2 \varepsilon_{1} \sin ^{2} \delta\left({ }^{3} D_{1}\right) \\
& \left.+3 \sqrt{2} \sin \left[2 \varepsilon_{1}\right] \sin \left[\delta\left({ }^{3} S_{1}\right)+\delta\left({ }^{3} D_{1}\right)\right]\right\},
\end{aligned}
$$

where notably there is no singlet phase-shift dependence. Our values of $\Delta$ are listed in Table I and plotted in Fig. 2 (solid dots). Clearly, on the average, our data are larger than predicted by PWA93 (solid curve).
The quantity $\varepsilon_{1}$ was determined from a single-energy, single-parameter $\chi^{2}$ minimization of $\left(\Delta_{\text {calc }}-\Delta_{\exp }\right)$ at each energy. $\Delta_{\text {calc }}$ was calculated from trial values of $\varepsilon_{1}$ and the remaining phase shifts were taken from PWA93. Using this procedure, the description of the cross-section and analyzing power data used in PWA93 remains virtually unchanged. The uncertainties associated with the triplet $n-p$ phase shifts given in Eq. (9) have a negligible influence on the overall uncertainty of $\varepsilon_{1}$. A $1 \%$ change in ${ }^{3} S_{1}$ at $E_{n}=10 \mathrm{MeV}$ changes the difference $\Delta$ by only $0.3 \%$. Modifications of the ${ }^{3} P_{j}$ phase-shift parameters that are consistent with the description of the $N N$ analyzing power data result in changes of $\Delta$ by only $0.1 \%$. Finally, a $5 \%$ change of ${ }^{3} D_{1}$ affects $\Delta$ by $0.25 \%$. This extremely small dependence of $\Delta$ on the triplet phase-shift parameters of Eq. (9) is an additional and unique advantage of our approach compared to earlier determinations of $\varepsilon_{1}$.

The results of the $\chi^{2}$ minimization of $\varepsilon_{1}$ are reported in Table I and plotted in the top panel of Fig. 3. The $20 \mathrm{MeV}$ value is from a $\chi^{2}$ minimization of $\Delta \sigma_{L}$ alone. Also shown in the figure are other published experimental $\varepsilon_{1}$ values [18-22] and theoretical predictions $[7,12,13,23]$. Our data support the trend indicated by the previous $\varepsilon_{1}$ determinations at $E_{n}=25$ and $50 \mathrm{MeV}$ and

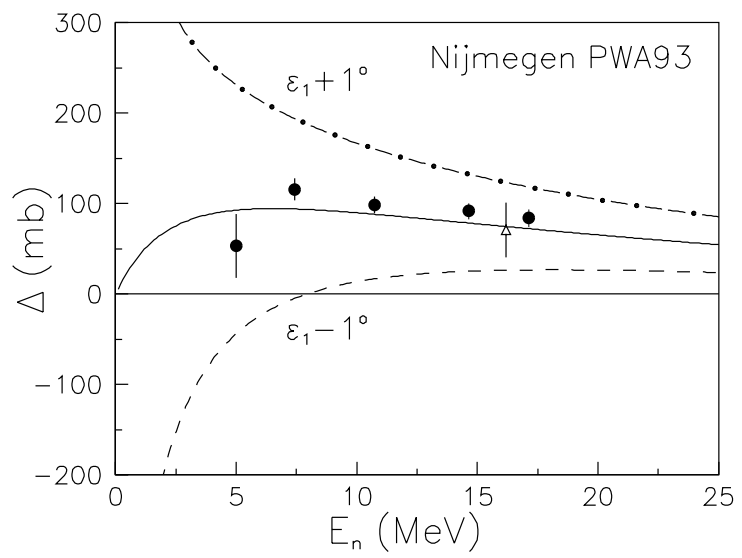

FIG. 2. Experimental results for the observable $\Delta=\Delta \sigma_{L}-$ $\Delta \sigma_{T}$. The error bars include statistical and systematic uncertainties. The datum at $E_{n}=16.2 \mathrm{MeV}$ (triangle) was obtained recently at Prague [18]. The curves show the sensitivity of $\Delta$ to changes in $\varepsilon_{1}$. The solid curve is the PWA93 prediction of $\Delta$, and the dot-dashed (dashed) curve is the predicted value of $\Delta$ with $\varepsilon_{1}$ varied by $+1^{\circ}\left(-1^{\circ}\right)$. See Eq. (9). 

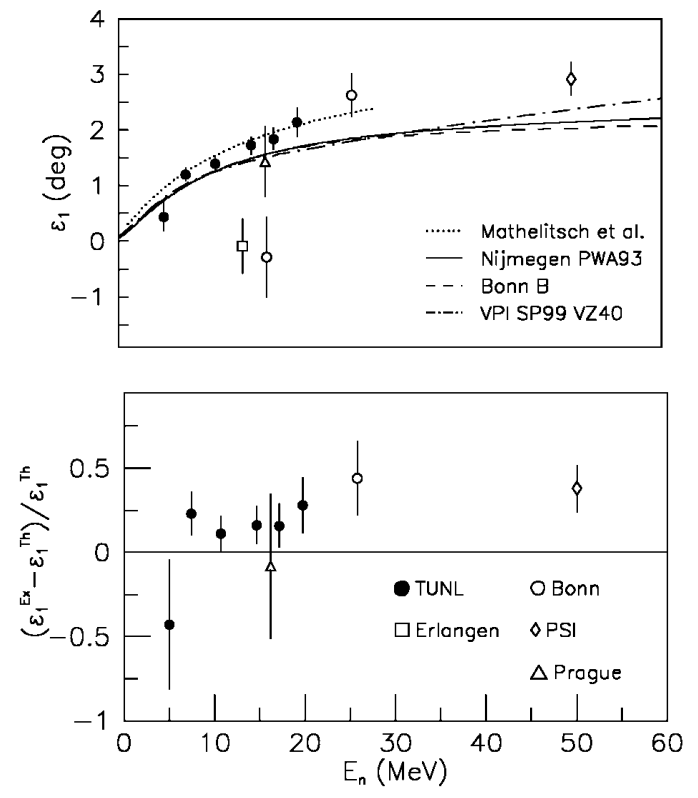

FIG. 3. Plot of $\varepsilon_{1}$ from present and previous experiments (top panel), and fractional deviation from the theoretical values of Nijmegen PWA93 (bottom panel). The previous data are

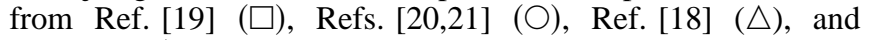

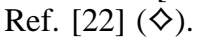

cast doubt on the accuracy of the approach used in $N N$ potential models. The results for $\varepsilon_{1}$ are higher in magnitude than the predictions of Nijmegen PWA93 (solid curve), VPI SP99 (0-400 MeV, dash-dotted curve), and the Bonn B $N N$ potential-model prediction [7] (dashed curve). On the other hand, the data agree well with predictions obtained from the effective-range parametrization of Mathelitsch and VerWest [13] (dotted curve). This parametrization invokes one pion exchange (OPE), but, contrary to the more recent $N N$ potential models and phase-shift analyses, here the OPE tensor force is not reduced by incorporating a $\pi N N$ form factor and the $\rho$-meson exchange contribution. In fact, our data and the deuteron properties can be described well by an OPE $N N$ potential with a charge dependent CD-Bonn type $\pi N N$ form factor, but without $\rho$ - and heavier meson-exchange contributions [24]. However, in order to fit the high-energy ${ }^{3} P_{0},{ }^{3} P_{2}$, and ${ }^{1} D_{2} N N$ phase shifts, a strong $\rho N N$ coupling is required [25]. Finally, our data are inconsistent with the two anomalously low $\varepsilon_{1}$ values near $E_{n}=15 \mathrm{MeV}[19,20]$. The bottom panel of Fig. 3 shows the relative deviation between the experimental data for $\varepsilon_{1}$ and the PWA93 prediction (here we excluded the two data points near $E_{n}=15 \mathrm{MeV}$ ). For energies above $E_{n}=5 \mathrm{MeV}$, the total $\chi^{2}$ is 21.8 .

In summary, we have performed polarized neutron beam-polarized proton target transmission measurements to determine the spin-dependent $n-p$ total cross-section differences $\Delta \sigma_{L}$ and $\Delta \sigma_{T}$. Single-energy, singleparameter phase-shift analyses were used to extract practically model insensitive values for the phase-shift parameter $\varepsilon_{1}$, which characterizes the strength of the $n-p$ tensor interaction at low energy. In combination with other measurements, our values of $\varepsilon_{1}$ support a $N N$ tensor interaction that is stronger than predicted by all modern ME based $N N$ potential models and the Nijmegen PWA93 and VPI SP99 analyses.

We gratefully acknowledge the support of the U.S. Department of Energy, Office of High Energy and Nuclear Physics, under Grants No. DEFG05-91-ER40619 and No. DEFG05-88-ER40441. We also acknowledge the support of The Robert A. Welch Foundation.

*Present address: Thomas Jefferson National Accelerator Facility, Newport News, VA 23606.

${ }^{\dagger}$ Present address: Avanti Corporation, Research Triangle Park, NC 27703.

*Present address: Los Alamos National Laboratory, Los Alamos, NM 87545.

[1] R. Machleidt, F. Sammarruca, and Y. Song, Phys. Rev. C 53, R1483 (1996).

[2] R. B. Wiringa, V. G. J. Stoks, and R. Schiavilla, Phys. Rev. C 51, 38 (1995).

[3] V.G. J. Stoks, R. A. M. Klomp, C.P.F. Terheggen, and J. J. de Swart, Phys. Rev. C 49, 2950 (1994).

[4] W. Glöckle, H. Witała, D. Hüber, H. Kamada, and J. Golak, Phys. Rep. 274, 107 (1996).

[5] Y. Wu, S. Ishikawa, and T. Sasakawa, Few-Body Syst. 15, 145 (1993).

[6] W. Tornow and H. Witała, Nucl. Phys. A637, 280 (1998).

[7] R. Machleidt, Adv. Nucl. Phys. 19, 175 (1989).

[8] T.B. Clegg et al., Nucl. Instrum. Methods Phys. Res., Sect. A 357, 200 (1995).

[9] A. Abragam and M. Goldman, Rep. Prog. Phys. 41, 395 (1978).

[10] B. van den Brandt, J. A. Konter, and S. Mango, Nucl. Instrum. Methods Phys. Res., Sect. A 289, 526 (1990).

[11] B.W. Raichle et al., in Application of Accelerators in Research and Industry, edited by J. L. Duggan and I. L. Morgan, AIP Conf. Proc. No. 475 (AIP, New York, 1999), pp. 223-226.

[12] V. G. J. Stoks, R. A. M. Klomp, M. C. M. Rentmeester, and J. J. de Swart, Phys. Rev. C 48, 792 (1993).

[13] L. Mathelitsch and B. J. VerWest, Phys. Rev. C 29, 739 (1984).

[14] B. W. Raichle, Ph.D. thesis, North Carolina State University, 1997.

[15] J. R. Walston, Ph.D. thesis, North Carolina State University, 1998.

[16] W.S. Wilburn et al., Phys. Rev. C 52, 2351 (1995).

[17] W. Tornow et al., in Physics with Polarized Beams and Polarized Targets, edited by J. Sowinski and S. Vigdor (World Scientific, Singapore, 1989), pp. 75-93.

[18] J. Brož et al., Z. Phys. A 359, 23 (1997).

[19] M. Schöberl et al., Nucl. Phys. A489, 284 (1988).

[20] P. Clotten et al., Phys. Rev. C 58, 1325 (1998).

[21] M. Ockenfels et al., Nucl. Phys. A526, 109 (1991).

[22] R. Henneck, Phys. Rev. C 47, 1859 (1993).

[23] R. A. Arndt, interactive computer code SAID, 1998.

[24] R. Machleidt (private communication).

[25] G. E. Brown and R. Machleidt, Phys. Rev. C 50, 1731 (1994). 